\title{
A case report pemphigoid gestational case, responding to systemic steroid therapy
}

Selma Bakar Dertlioğlu

Associate Professor of Dermatology, University of Health Sciences,Training and Research Hospital, Department of Dermatology, 23100 Elazig, Turkey.

Corresponding Author: Selma Bakar Dertlioğlu, MD, Associate Professor of Dermatology, University of Health Sciences, Training and Research Hospital, Department of Dermatology, 23100 Elazig, Turkey.

Received date: January 16, 2021: Accepted date: February 25, 2021: Published date: March 11, 2021

Citation:Selma B Dertlioğlu (2021) A case report pemphigoid gestational case, responding to systemic steroid therapy J Clinical Cas Rep and stud 2(2); DOI: 10.31579/2690-8808/060

Copyright: (C) 2021, Selma Bakar Dertlioğlu. This is an open access article distributed under the Creative Commons Attribution License, which permits unrestricted use, distribution, and reproduction in any medium, provided the original work is properly cited.

\begin{abstract}
In this case report, a rare case of Pemphigoid Gestationis at 33 weeks' of gestation is presented and the related literature is briefly reviewed. A 21 -year-old at 33 weeks' of gestation admitted with the complain of itchy skin lesions on the abdomen, arms and legs. Dermatologic examination revealed urticaria papules and plaques in the abdomen, arms and legs, more intensely around the umbilicus, which range from 1-2 cm diameter to $4-5 \mathrm{~cm}$ diameter. Papulovesicular lesions and few erosions were observed especially in the hand and foot dorsals.

Spongiosis on epidermis, intracellular and subepidermal bullae formation, edema on papillary architecture and inflammatory infiltrate composed of mononuclear cells and eosinophils around the perivascular ares of the upper dermis were seen in histological examination. Direct immunofluorescence of perilesional skin showed linear deposition of complement (C3) along the basement membrane zone. The preliminary diagnosis of pemphigoid gestationis was confirmed by histopathologic findings.

Pemphigoid gestationis is a rare autoimmune bullous dermatosis that begins in the second or third trimester of pregnancy. Healing occurs weeks after birth. It occurs more severely in subsequent pregnancies. Its main treatment is systemic steroid and antihistamine administration.

A multidisciplinary approach both in dermatological and gynecological terms is required for the diagnosis, treatment and course of the disease.
\end{abstract}

Keywords: steroid therapy; Pemphigoid Gestationis; Dermatologic examination; papillary architecture;

\section{Introduction}

Pemphigoid gestation (PG) is clinically presents with severe pruritus that precedes the appearance of vesiculobullous and target-like lesions, which tend to form groups on erythematous-urticarial grounds, and show anular and scaly lesions. It is a pregnancy dermatosis showing the clinical and histopathological features of the bullous pemphigoid. Mucosal lesions and facial involvement are rare [1]. The disease is originally called Herpes gestationes (HG) because of the herpetiform appearance of vesicles and bullae. Despite its name, HG is not associated with herpetic infection [2].

\section{Case Presentation}

We were consulted by a 33-year-old pregnant woman at the age of 21, who applied to the dermatology outpatient clinic due to itchy skin lesions in the abdomen, arms, legs, hands and feet. In her dermatological examination; Urticaria papules and plaques on the abdomen, arms and legs, more intensely around the umbilicus, which ranged from $1-2 \mathrm{~cm}$ diameter to $4-5 \mathrm{~cm}$ diameter, papulovesicular lesions in the hands and feet, lesions in the target area, and small number of erosions were seen (Figure 1,2,3,4). 


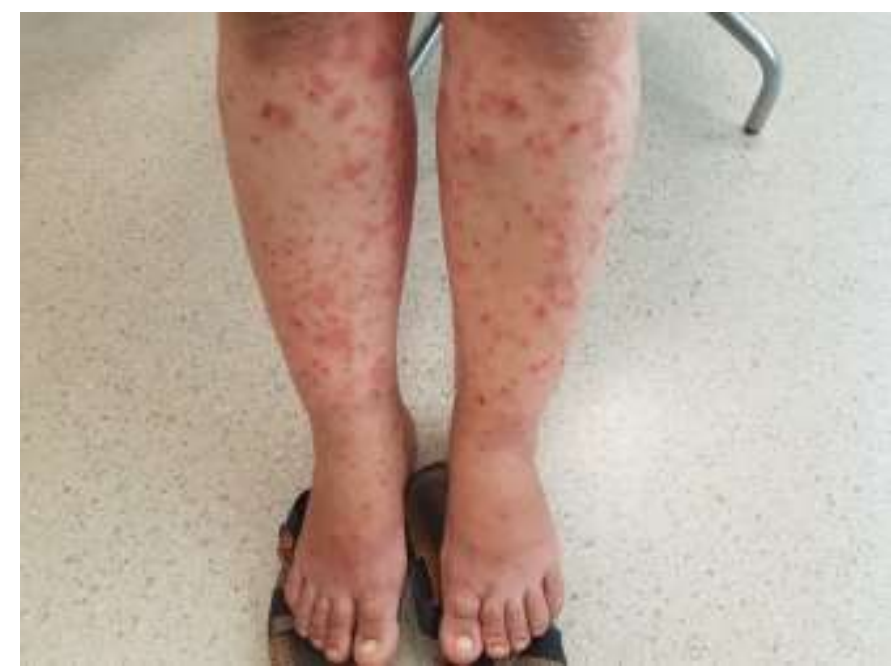

Figure 1. Urticaria papules and plaques on the abdomen, arms and legs, more intensely around the umbilicus, which ranged from 1-2 $\mathrm{cm}$ diameter to 4-5 cm diameter, papulovesicular lesions in the hands and feet, lesions in the target area, and small number of erosions

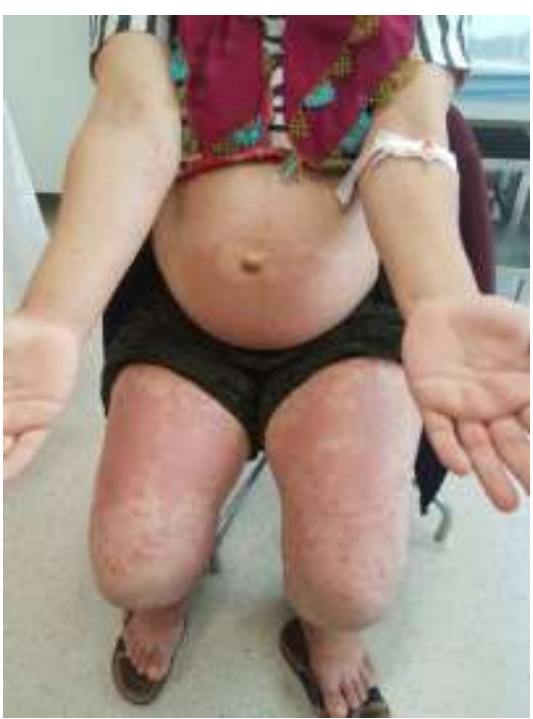

Figure 2. Urticaria papules and plaques on the abdomen, arms and legs, more intensely around the umbilicus, which ranged from 1-2 $\mathrm{cm}$ diameter to 4-5 cm diameter, papulovesicular lesions in the hands and feet, lesions in the target area, and small number of erosions 


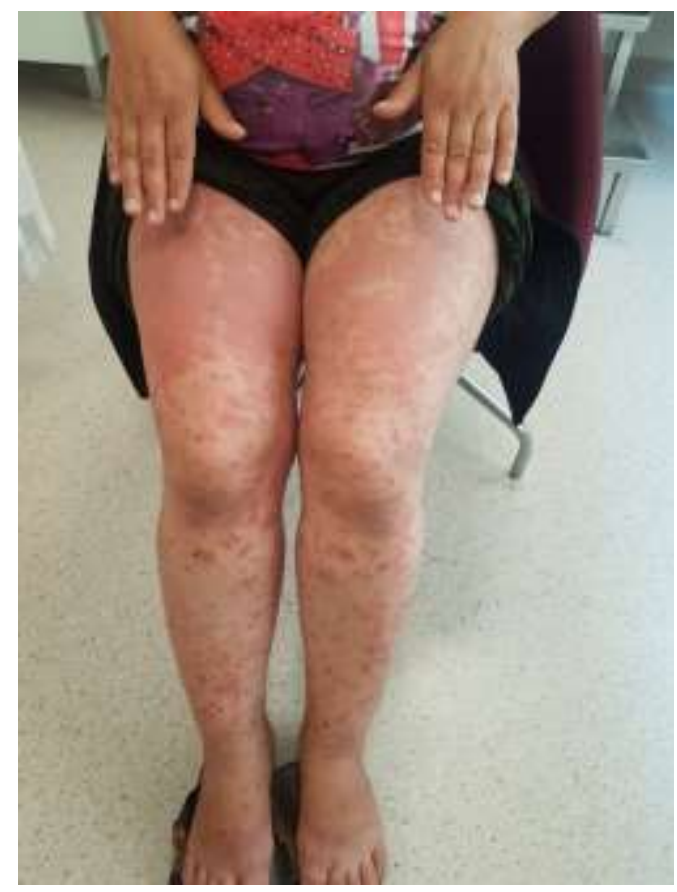

Figure 3. Urticaria papules and plaques on the abdomen, arms and legs, more intensely around the umbilicus, which ranged from 1-2 $\mathrm{cm}$ diameter to 4-5 cm diameter, papulovesicular lesions in the hands and feet, lesions in the target area, and small number of erosions

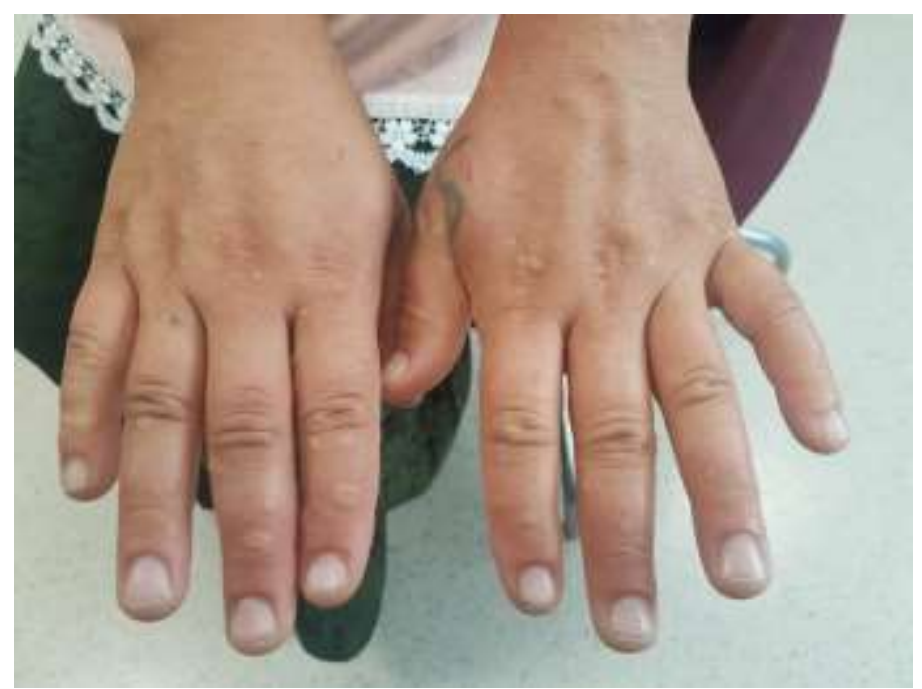

Figure 4. Urticaria papules and plaques on the abdomen, arms and legs, more intensely around the umbilicus, which ranged from 1-2 $\mathrm{cm}$ diameter to 4-5 cm diameter, papulovesicular lesions in the hands and feet, lesions in the target area, and small number of erosions

Mucosal lesion was not observed. The patient medical history was unremarkable, and she denied use of any other medications or herbal remedies at the time the symptoms started or since. Laboratory tests revealed leukocytosis, eosinophilia, neutrophilia and increased erythrocyte sedimentation. The thyroid disease in which the most common association was reported was not detected in our case.

For histopathological evaluation, a perilesional incisional punch biopsy was performed from the lesioned area. In the routine histopathological examination; subepidermal separation and bulla formation, mixed type inflammatory cell infiltration rich in eosinophilic leukocytes in and around the bulla and perivascular areas, epidermal eosinophilic spongiosis around the separation and edema in the papillary dermis were observed (Figure 5,6). Direct immunofluorescence of perilesional skin showed linear deposition of complement (C3) along the basement membrane zone of the epidermis. 


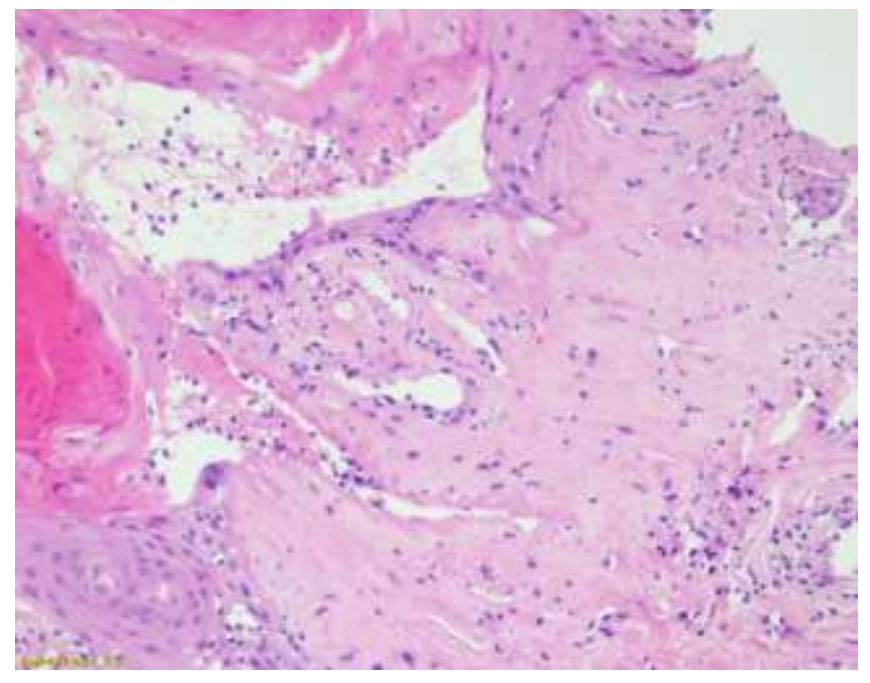

Figure 5: Spongiosis, bulla formation in the epidermis and perivascular inflammatory cell infiltration in the superficial dermis. H\&E X100

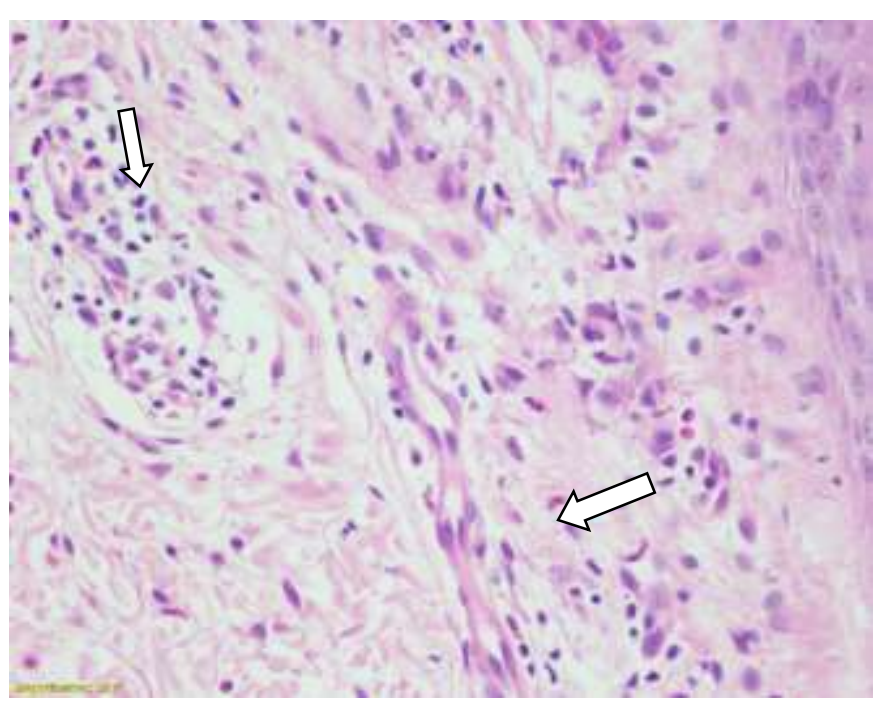

Figure 6: Inflammatory infiltrate consisting of eosinophils in the perivascular area. H\&E X400

With these clinical and histopathological findings, the patient was diagnosed with herpes gestation. Wet dressing, antihistamines, topical corticosteroids were started, but systemic prednisolone $(0.5 \mathrm{mg} / \mathrm{kg} /$ day $)$ treatment was initiated for the patient due to the severity of the lesions [1]. Prednisolone dose of the patient, whose lesions regressed during follow-up, was decreased gradually. The patient, who did not experience any problems during the remaining pregnancy follow-up, had a normal spontaneous delivery at term. No pathology was found in the newborn examination. Postpartum follow-up went smoothly.

\section{Discussion}

Pemphigoid gestationis (PG) is a rare vesiculobullous dermatosis of pregnancy characterized by its self-limited course. It is a condition more commonly seen in multiparous females over primiparous females. PG starts typically in the second or third trimester mean onset at 21 to 28 weeks gestation). PG usually recurs in subsequent pregnancies with early onset and increased severity. Most fetuses are unaffected; however, transient lesions occur in $<5 \%$ of neonates born to mothers with pemphigoid gestationis. Risks, including infant mortality, are increased after premature delivery and in infants who are small for gestational age $[3,4]$. PG has also been reported in association with trophoblastic tumors, such as choriocarcinoma [5].
The cutaneous manifestations of PG consist of pruritic papules and urticarial plaques with an erythematous base which evolve to vesicle or tense bullae. Pruritus may precede the skin lesions. PG often begins in the umbilical region before spreading to the rest of the abdomen and thighs. It can progress to involve the entire skin's surface including palms and soles. The face and the mucous membranes are usually not affected. Flare-ups are common 24 to 48 hours postpartum and can occur during subsequent menses or ovulation [3, 4].

Routine histology is also a fundamental part of the diagnosis showing a subepidermal vesicle with a perivascular lymphocytic infiltrate with numerous eosinophils. Eosinophils may appear at the dermoepidermal junction and in the blister cavity. Immunohistopathologic studies confirm the diagnosis of PG. Direct immunofluorescence of perilesional skin, using salt-split skin, is the gold standard which shows linear C3 deposition along the dermal-epidermal junction in all cases and IgG deposits in some cases [6].

Treatment varies depending on the stage and severity of the disease. The goal is to control symptoms and to prevent blister formation. Symptomatic treatment includes topical emollients with oatmeal baths and systemic $\mathrm{H} 1$ antihistamine such as diphenhydramine or chlorpheniramine. Actions such as washing clothing and bedding in mild 
detergent along with mild bathing soaps are beneficial. Still, some patients require systemic corticosteroids to control the pruritus. In rare cases, more aggressive immunosuppressive therapy may be needed to save the life of the mother or the fetus [7].

\section{Conclusion}

Pregnant women diagnosed with PG should be considered in the risky group. Potential fetal and maternal risks should be discussed with the family. Obstetrics, dermatology and pediatricians should work together to prevent and treat maternal, fetal and neonatal complications.

\section{References}

1. Singla A, Shree S, Mehta S. (2016) Pregnancy with Pemphigoid Gestationis: A Rare Entity. Journal of Clinical and Diagnostic Research. 10(7): 6-7.

2. Baksu B, Gökdemir G, Çınar S, Davas D, Akyol A. (2004) Yirmiüç haftalık gebelikte herpes gestasyones: Bir olgu sunumu. Perinatoloji Dergisi. 12: 183-6.

3. Dermatologic Disorders. In: Cunningham FG, MacDonald PC, Gant NF, Leveno KJ, Gilstrap LC, Hankins GDV, Clark SL, eds. (1997) Williams Obstetrics. 20th ed. Conneticut, Appleton and Lange. p.1273-9.

4. Kroumpouzos G, Cohen LM. (2003) Specific dermatoses of pregnancy: An evidence-based systematic review. Am J Obstet Gynecol. 188: 1083-90.

5. Semkova K, Black M. (2009) Pemphigoid gestationis: current insights into pathogenesis and treatment. Eur J Obstet Gynecol Reprod Biol. 145(2): 138-44.

6. Bedocs P.M. Kumar V. Mahon M.J. (2009) Pemphigoid gestationis: a rare case and review. Arch Gynecol Obstet. 279: 235-8.

7. Cohen S, Strowd LC, Pichardo RO. (2018) Pemphigoid gestationis: a case series and review of the literature. $J$ Dermatolog Treat. 29(8): 815-8. 